\title{
Label-free oligonucleotide biosensor based on dual-peak long period fiber grating
}

\author{
Xianfeng Chen*a, Chen Liu ${ }^{\mathrm{a}}$, Marcos R. Cardoso ${ }^{\mathrm{b}}$, Cleber R. Mendonça ${ }^{\mathrm{b}}$, \\ David A. Nagel ${ }^{c}$, Anna V. Hine ${ }^{c}$, Lin Zhang ${ }^{d}$ \\ ${ }^{a}$ School of Electronic Engineering, Bangor University, United Kingdom; \\ ${ }^{\mathrm{b}}$ Instituto de Física de São Carlos, Universidade de São Paulo, Brazil; \\ ${ }^{\mathrm{c}}$ School of Life and Health Sciences, Aston University, United Kingdom; \\ ${ }^{\mathrm{d}}$ Aston Institute of Photonic Technologies, Aston University, United Kingdom
}

\begin{abstract}
We report the simplification and development of biofunctionalization methodology based on one-step 1-ethyl-3-(3dimethylaminopropyl)carbodiimide (EDC)-mediated reaction. The dual-peak long period grating (dLPG) has been demonstrated its inherent ultrahigh sensitivity to refractive index (RI), achieving 50 -fold improvement in RI sensitivity over a standard LPG sensor used in low RI range. With the simple and efficient immobilization of unmodified oligonucleotides on sensor surface, dLPG-based biosensor has been used to monitor the hybridization of complementary oligonucleotides showing a detectable oligonucleotide concentration of $4 \mathrm{nM}$ with the advantages of label-free, real-time, and ultrahigh sensitivity.
\end{abstract}

Keywords: Optical fiber biosensor; Long period grating; Label-free; Oligonucleotide; DNA; EDC-mediated reaction

\section{INTRODUCTION}

Optical fibers exhibit the inherent advantages of compact size, light weight and electromagnetic interference immunity. The traditional biosensors based on culture collection and colony counting were complicated, hazardous, expensive, time consuming and may require signal amplification or labeling. Over the last decade, the biosensors based on optical fibers have attracted great attention and made rapid advances ${ }^{1}$. Fiber optic sensor can provide higher sensitivity and selectivity than the traditional sensors ${ }^{2,3}$.

To date various fiber optic biosensors have been developed by using long period gratings (LPGs), tilted fiber gratings (TFGs), micro fiber Bragg gratings (mFBGs) ${ }^{4-8}$. It has been reported that the maximum refractive index sensitivity of the LPG can be achieved when the surrounding refractive index (RI) approaches the value of the fiber cladding ${ }^{9}$. This is far from the RI range 1.33-1.35 RIU (refractive index unit) of aqueous solutions where bioassays and biochemical events are typically carried out. Several approaches have been investigated to improve the sensitivity of the LPGs, such as etching the cladding, polishing the side and tapering the fiber. However, those approaches become more complex and increase cost. In contrast, a unique property of the dual-peak LPG (dLPG) has been revealed whereby a set of dispersion-turningpoints exists in LPG phase-matching curves, resulting in the conjugate cladding modes observed as dual-peak features in the transmission spectrum ${ }^{4,10}$. Since they are in close proximity to the dispersion-turning-point, the dual-peak cladding modes are extremely sensitive to the surrounding-medium refractive index (SRI) change. To date, various fiber optic biosensors have been developed for the detection of antibody-antigen interactions, biotin-streptavidin, DNA hybridization, enzyme-glucose binding and cellular behavior ${ }^{5,7,11}$.

In this paper, we report one-step 1-ethyl-3-(3-dimethylaminopropyl)carbodiimide (EDC) -mediated reaction for the immobilization of oligonucleotides on dLPG-based biosensors. The simplification of biomolecule immobilization procedure is feasible by using only EDC as the heterobifunctional cross-linker for 5' -phosphorylated oligonucleotides. The biofunctionalized dLPG has been used to detect the hybridization of complementary oligonucleotides in real-time, showing a lower limit of detection and high sensitivity.

*x.chen@bangor.ac.uk; phone 441248 382480; fax 441248361429

Optical Fibers and Sensors for Medical Diagnostics and Treatment Applications XVI, edited by Israel Gannot Proc. of SPIE Vol. 9702, 97020S · (c) 2016 SPIE · CCC code: 1605-7422/16/\$18 · doi: 10.1117/12.2207869 


\section{DUAL-PEAK LPG FABRICATION AND REFRACTIVE INDEX SENSITIVITY}

\subsection{Inscription of dual-peak LPG}

LPG couples the light from the core mode to co-propagating cladding modes resulting in the transmission spectrum containing a series of attenuation bands. Each attenuation band corresponds to the coupling to a cladding mode satisfying the phase-matching condition ${ }^{12}$ :

$$
\lambda_{\text {res }}=\left(n_{c o}^{e f f}-n_{c l, m}^{e f f}\right) \Lambda
$$

where $\Lambda$ is the grating period, $n_{c o}^{\text {eff }}$ and $n_{c l, m}^{\text {eff }}$ are the effective indices of core and $\mathrm{m}^{\text {th }}$ cladding mode. It has been reported that a set of dispersion-turning-point exists on the phase-matching curves due to the parabolic property of the group index of the higher cladding modes ${ }^{4}$. With a selected relatively short LPG period, the light coupling between the fundamental core mode and a higher order cladding mode leads to two attenuation peaks in the transmission spectrum with respect to the conjugate cladding mode. LPGs with such dual-peak features are defined as dual-peak LPGs.

The dLPGs with length of $3 \mathrm{~cm}$ and period of $157 \mu \mathrm{m}$ were UV inscribed in hydrogenated SMF28 fiber by a CW frequency-doubled Ar laser (244nm) using the point-by-point fabrication method. After UV inscription, the gratings were annealed at $80^{\circ} \mathrm{C}$ for $48 \mathrm{~h}$ to remove the residual hydrogen and to stabilize their optical properties. During the UV inscription process, the dLPG spectra were recorded (Fig. 1). Two resonances initially appeared at $1180 \mathrm{~nm}\left(L P_{012}^{\text {blue }}\right)$ and $1630 \mathrm{~nm}\left(L P_{012}^{\text {red }}\right)$, moved towards each other with multiple scan. With further UV exposure, the dual peaks moved more closer and became stronger, whereas a lower mode $\left(\mathrm{LP}_{011}\right)$ peak at $1010 \mathrm{~nm}$ appeared and moved only a small red-shift.

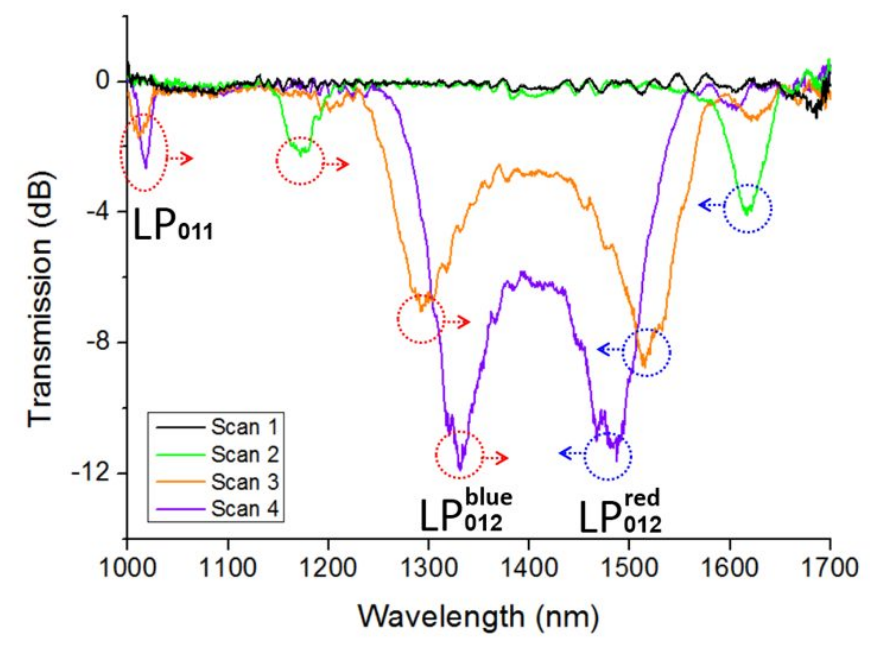

Figure 1. Dual-peak LPG spectral evolution under UV exposure during inscription.

\subsection{Refractive index property of dual-peak LPG}

To study the RI sensitivity, the dLPG was immersed in a set of index gels and the spectrum was measured for each RI value. The wavelength shift of dLPG resonances were plotted in Fig. 2. Over the range of RI from 1.30 to 1.44, the redpeak $L P_{012}^{r e d}$ was further red-shifted with increasing RI whereas the blue-peak $L P_{012}^{\text {blue }}$ was further blue-shifted. In contrast, the $\mathrm{LP}_{011}$ peak showed only a slight blue-shift with increasing RI. It can be seen from Fig. 2 that the resonances moved nonlinearly with an increasing trend over the whole RI range. The total wavelength shifts of $\mathrm{LP}_{011}, L P_{012}^{\text {blue }}$ and $L P_{012}^{r e d}$ are $-10.6 \mathrm{~nm},-111.7 \mathrm{~nm}$, and $+225.0 \mathrm{~nm}$ respectively (with a calculated estimation for the final red-peak which had moved out of the detectable range). The detail of RI characteristics for different peaks are compared, it is particularly noteworthy that both the blue and red peaks show an ultrahigh RI sensitivity of $-2914 \mathrm{~nm} / \mathrm{RIU}$ and $+7488 \mathrm{~nm} / \mathrm{RIU}$ in the high RI range (1.432 to 1.440), respectively, whereas the $\mathrm{LP}_{011}$ peak has a low sensitivity of $-288 \mathrm{~nm} / \mathrm{RIU}$. The blue and 
red peaks also show high RI sensitivity of $-564 \mathrm{~nm} / \mathrm{RIU}$ and $+794 \mathrm{~nm} / \mathrm{RIU}$, respectively, in the low RI range (1.30 to 1.35) - the range in which bioassays and biochemical event-monitoring are usually carried out. The maximum RI sensitivity (794 nm/RIU) in low range RIs of 1.30-1.35 of our dLPG is more than 50 times higher than that of previously reported $370 \mu \mathrm{m}$-period LPG sensor, which achieved an RI sensitivity of $15 \mathrm{~nm} / \mathrm{RIU}$ around $1.33 \mathrm{RIU}^{11}$. Hence, biosensors based on dLPGs should achieve comparable or better performances with respect to the standard LPG or mFBG based biosensors ${ }^{8,11}$.

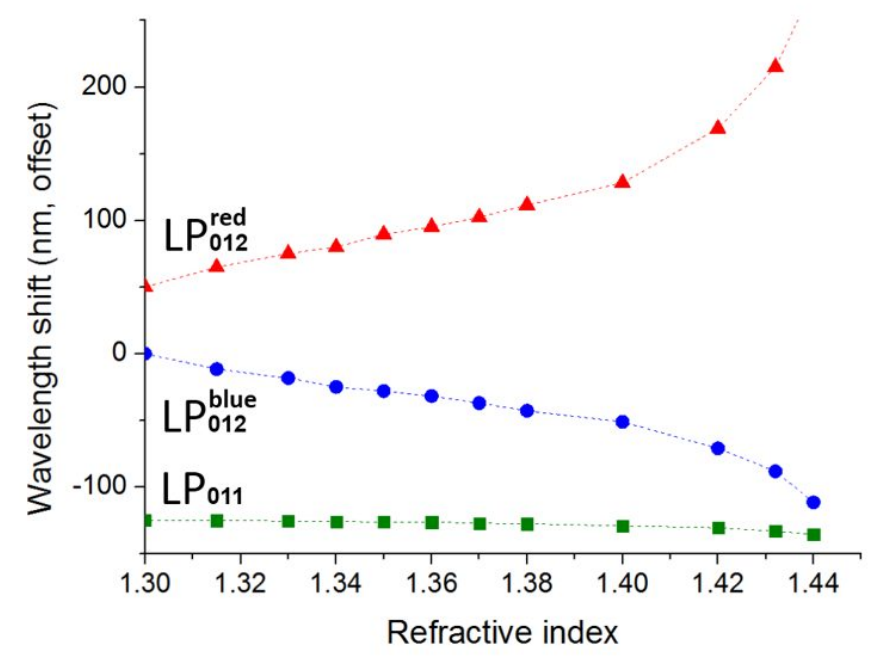

Figure 2. Wavelength shifts of dual-peak LPG resonances against the refractive index changes of surrounding medium.

\section{BIOFUNCTIONALIZATION OF DUAL-PEAK LPG}

It is important to immobilize the probe biomolecules on the sensor surface to generate a fiber optic biosensor. Fig. 3 depicts the generation of fiber biosensor from the bare glass fiber to probe oligonucleotide immobilization.

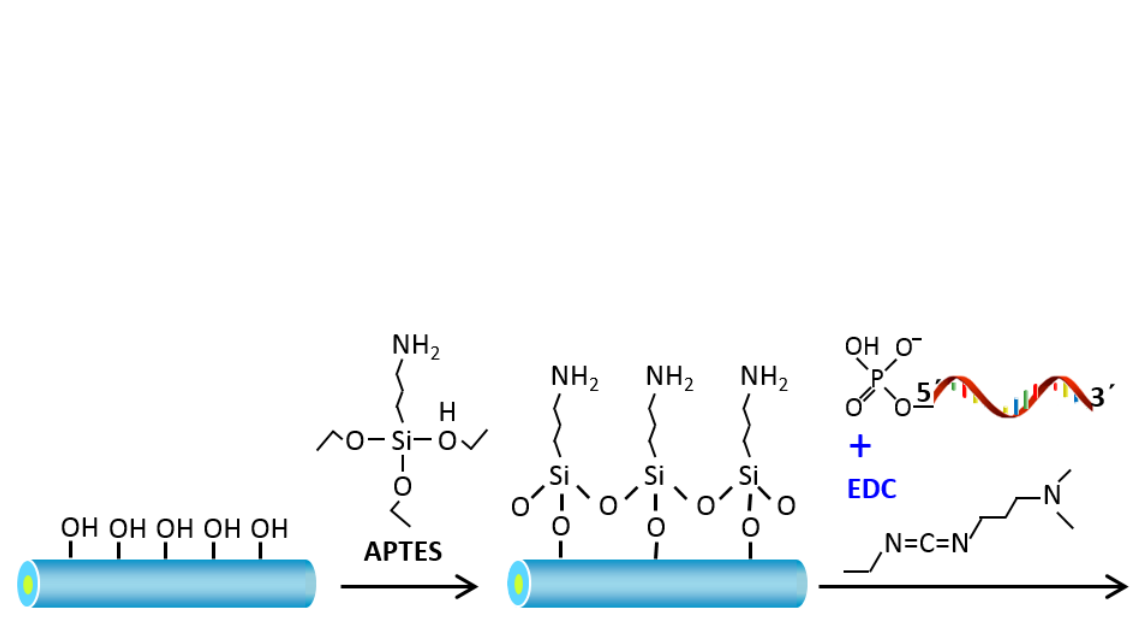

(a)

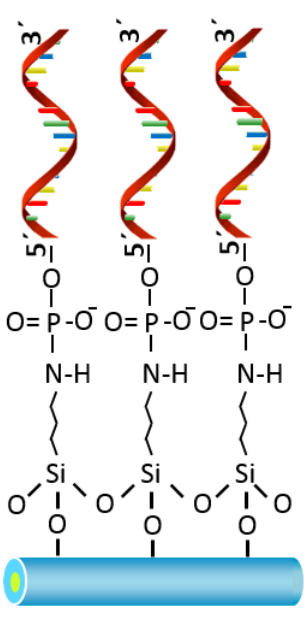

(c)

Figure 3. Schematic diagram of biofunctionalization of fiber optic biosensor: (a) Cleaned glass fiber surface.

(b) Silanization of glass fiber surface by APTES. (c) Covalent immobilization of 5 '-phosphorylated oligonucleotides via EDC-mediated reaction. 
The glass fiber in the region of dLPG was soaked in freshly-prepared $5 \mathrm{M} \mathrm{HCl}$ solution for $30 \mathrm{~min}$, thoroughly rinsed with deionized (DI) water then dried in the air (Fig. 3a). It was then immersed in a fresh, 10\% solution of (3Aminopropyl)triethoxysilane (APTES) for 50 min generating surface primary amine groups (Fig. 3b). The fiber sensor was then rinsed with DI water and again air-dried. An aqueous solution of 5'-phosphorylated oligonucleotide (5'phosphate- GCA CAG TCA GTC GCC -3') was diluted with $30 \mathrm{mM} 1$-methylimidazole to a final concentration of $5 \mu \mathrm{M}$, and $10 \mathrm{mg} / \mathrm{ml} \mathrm{EDC}$ was added and mixed for $1 \mathrm{~min}$. The amino-silanized glass fiber was immediately immersed into this oligonucleotide solution, which was placed in a closed container to avoid evaporation, and incubated overnight at room temperature. After $16.5 \mathrm{~h}$ incubation, the glass fiber was thoroughly rinsed in DI water, dried in the air and stored in dry container at room temperature for further use. The oligonucleotide was now covalently-linked to the biosensor (Fig. 3c) and available for hybridization to a complementary DNA strand. All the biochemical experiments were performed in a fume cupboard. To minimize the bend cross-sensitivity, the fiber grating sensors were placed straight in a custom-made V-groove container on a Teflon plate and all the chemicals and solvents were added and withdrawn from the container by careful pipetting.

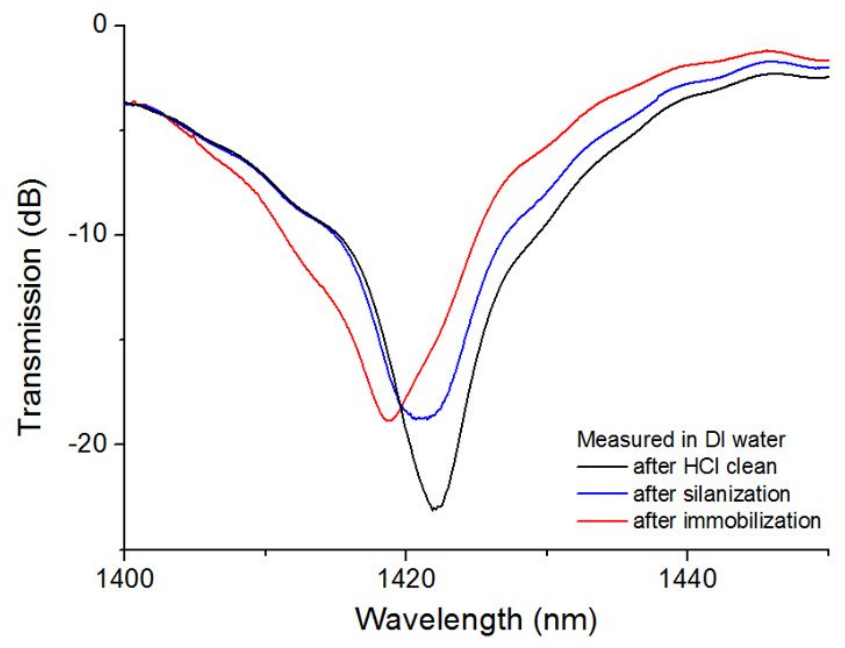

Figure 4. Spectra captured in DI water after cleaning, silanization and oligonucleotide immobilization.

The biofunctionalization of the dLPG sensor was determined by monitoring the spectral evolution of dLPG in situ throughout the cleaning, silanisation and immobilization processes. A $3 \mathrm{~cm}$-long dLPG with a period of $158.5 \mu \mathrm{m}$ was used for this experiment. The blue-peak at $1422 \mathrm{~nm}$ (in DI water) was selected for the following experiments. Fig. 4 shows the transmission spectra of this peak, captured in DI water, after $\mathrm{HCl}$ cleaning, APTES silanization, and oligonucleotide immobilization, respectively. A clear blue-shift has occurred in response to this biofunctionalization procedure. Specifically, the silanization has induced a relatively small blue-shift of $-1024 \mathrm{pm}$ whereas the immobilization has moved the peak by a further $-2320 \mathrm{pm}$. Clearly, the APTES- modification and the attachment of $5^{\prime}-$ phosphorylated oligonucleotides on the fiber surface have each changed the effective index of cladding, so demonstrating successful immobilization.

\section{LABEL-FREE BIODETECTION}

The dLPG device immobilized with oligonucleotide was used to detect the hybridization of complementary oligonucleotide (Fig. 5). The optical spectral evolution during the hybridization process was monitored by the use of the optical interrogation system where the broadband source launched the light into the dLPG biosensor whose spectrum was monitored by optical spectrum analyzer. Following DNA immobilization, the dLPG sensor was washed in DI water and rinsed in $6 \times$ SSPE buffer $(0.9 \mathrm{M} \mathrm{NaCl}, 0.06 \mathrm{M} \mathrm{NaH} 2 \mathrm{PO} 4$, and $0.006 \mathrm{M}$ EDTA) for $10 \mathrm{~min}$, then immersed in fresh $2 \mu \mathrm{M}$ complementary oligonucleotide (5'- phosphate- GCA CAG TCA GTC GCC -3 ') in 6×SSPE buffer and allowed to react at room temperature for $60 \mathrm{~min}$. 


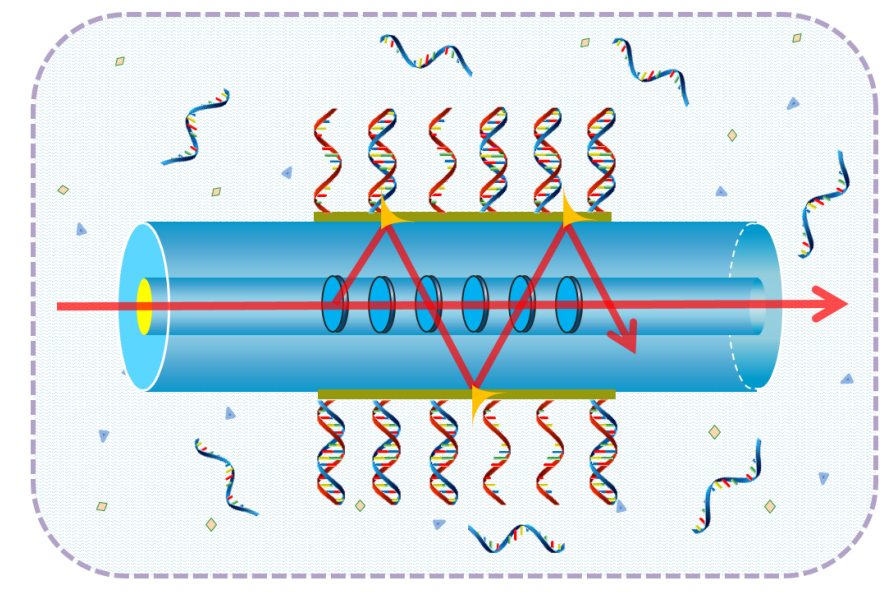

Figure 5. Hybridization process between probe oligonucleotide immobilized on dLPG and complementary oligonucleotides.

The blue-peak $L P_{012}^{\text {blue }}$ described above was monitored in situ throughout the whole hybridization process. As can be seen from Fig. 6, the resonant peak shows a blue-shift of $-1044 \mathrm{pm}$ over the whole hybridization process. There are three stages associated with the 60 min DNA hybridization. A rapid reaction occurs in the first 3 min with the wavelength shift of $-490 \mathrm{pm}$, which equates to a detection sensitivity of $245 \mathrm{pm}$ blue-shift per $\mu$ M DNA (or $4 \mathrm{nM}$ DNA per pm blueshift). This initial reaction is followed by a steady process ( 3 to $20 \mathrm{~min}$ ) with the wavelength shift of $-216 \mathrm{pm}$, and finally a much slower reaction (20 to $60 \mathrm{~min}$ ) with the shift of $-338 \mathrm{pm}$. Thus maximum sensitivity occurs in the first 3 mins. Although not described herein, it is therefore feasible that the dLPG sensor could be used to study binding kinetics. The sensor measurements are rapid (503 ms per full scan for the selected 'blue-peak') and in the current study, measurements were taken every $30 \mathrm{~s}$ for the first 20 mins. However, the measurement time could easily be reduced to $1 \mathrm{scan} / \mathrm{s}$ and the resulting data used to give kinetic analyses of initial rates of binding. Finally, if the noise effect could be eliminated effectively by using an optical interrogation system with $1 \mathrm{pm}$ resolution, this would theoretically lead to a detection sensitivity of $4 \mathrm{nM}$ oligonucleotide concentration, which would be two orders of magnitude lower than the microfiber Bragg grating based DNA biosensor ${ }^{8}$.

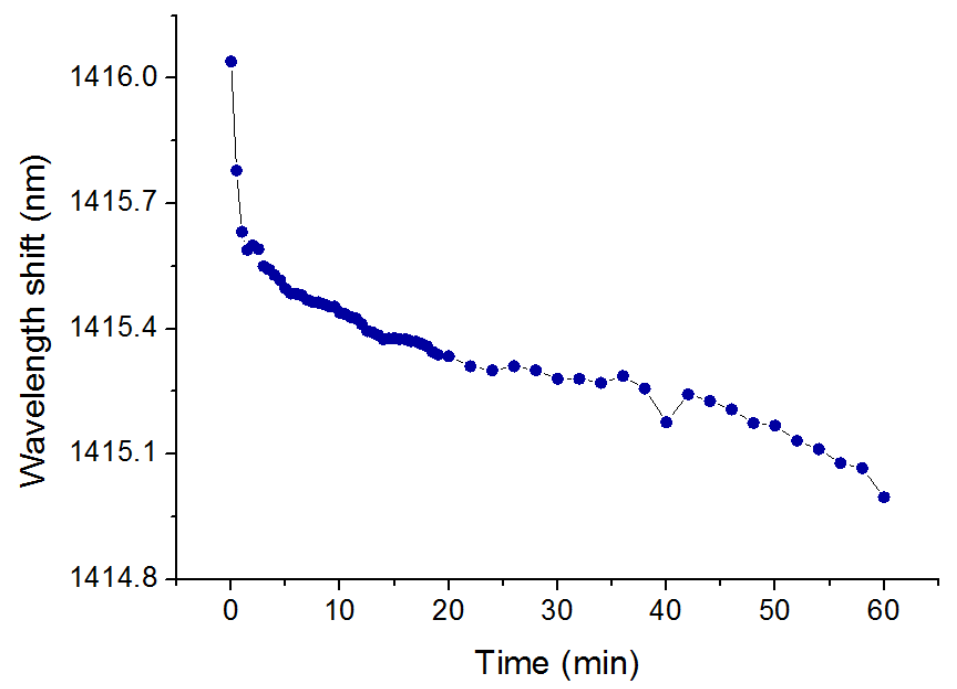

Figure 6. Wavelength shift against time during the DNA hybridization process. 


\section{CONCLUSION}

In this work, a dual-peak LPG based biosensor has been developed to detect the hybridization of oligonucleotides in realtime. Moreover, a one-step EDC-mediated procedure has been demonstrated to facilitate the covalent immobilization of 5 '-phosphorylated oligonucleotide on an amino-modified glass fiber sensor surface. The dual-peak LPG with inherent ultrahigh / high RI sensitivity has been demonstrated to detect a quantifiable optical signal corresponding to the refractive index change of the bio-analytes in which the biological recognition events occurred. After functionalization, the dLPG biosensor was used to monitor the hybridization of complementary oligonucleotides showing a detectable oligonucleotide concentration of $4 \mathrm{nM}$. The proposed one-step EDC reaction approach can be further extended to develop fiber optic biosensors for disease analysis and medical diagnosis with the advances of label-free, real-time and high sensitivity.

\section{ACKNOWLEDGEMENTS}

This work was supported by Marie Curie Scheme included in the $7^{\text {th }}$ Framework Programme of the European Union (FP7 PIRSES-2013-612267, iPhoto-Bio). The authors also acknowledge the support from the Sêr Cymru NRN.

\section{REFERENCES}

[1] Fan, X. and White, I.M. 2011. Nature Photonics, 5, 591-597. Optofluidic microsystems for chemical and biological analysis.

[2] Canning, J., 2009. J of Sensors Vol. 2009, 871580, 17 pages. Properties of specialist fibres and Bragg gratings for optical fibre sensors.

[3] Cusano, A., Consales, M., Crescitelli, A., Ricciardi, A. (Eds.) 2014. Springer. Lab-on-Fiber Technology.

[4] Shu, X., Zhang, L., Bennion, I., 2002. J. Lightwave Technol. 20, 255-266. Sensitivity characteristics of longperiod fiber gratings.

[5] Chen, X., Zhang, L., Zhou, K., Davies, E., Sugden, K., Bennion, I., Hughes, M., Hine, A. 2007. Opt. Lett. 32, 2541-2543. Real-time detection of DNA interactions with long-period fiber-grating-based biosensor.

[6] Albert, J., Shao, L.-Y., Caucheteur, C. 2013. Laser Photonics Rev., 7: 83-108. Tilted fiber Bragg grating sensors.

[7] Luo, B., Yan, Z., Sun, Z., Li, J., Zhang, L. 2014. Opt. Express 22, 30571-30578. Novel glucose sensor based on enzyme-immobilized $81^{\circ}$ tilted fiber grating.

[8] Sun, D., Guo, T., Ran, Y., Huang, Y., Guan, B.O. 2014. Biosens Bioelectron. 61, 541-546. In-situ DNA hybridization detection with a reflective microfiber grating biosensor.

[9] Patrick, H., Kersey, A., Bucholtz, F. 1998. J. Lightwave Technol. 16, 1606-1611. Analysis of the response of long period fiber gratings to external index of refraction.

[10] Smietana, M., Koba, M., Mikulic, P., Bock, W.J. 2014. Opt. Express 22, 5986-5994. Measurements of reactive ion etching process effect using long-period fiber gratings.

[11]Chiavaioli, F., Trono, C., Giannetti, A., Brenci, M., Baldini, F. 2014. J. Biophotonics, 7, 312-322. Characterisation of label-free biosensor based on long period grating.

[12] Vengsarkar, A., Lemaire, P., Judkins, J., Bhatia, V., Erdogan, T., Sipe, J. 1996. J. Lightwave Technol. 14, 5864. Long-period fiber gratings as band-rejection filters. 